\title{
Improvements and Shortcomings in Emergency Oxygen Prescribing: A Quality Improvement Initiative at an Acute Tertiary Care Hospital
}

\author{
Authors: \\ *Hoda Ranjbar, Shruti Dorai \\ The Royal Sussex County Hospital (RSCH), Brighton and Sussex University Hospital \\ NHS Trust, Brighton, East Sussex, UK \\ *Correspondence to h.ranjbar@nhs.net \\ Disclosure: $\quad$ The authors have declared no conflicts of interest. \\ Received: $\quad 26.05 .20$ \\ Accepted: $\quad 02.02 .21$ \\ Keywords: $\quad$ Acute, chronic pulmonary obstructive disorder (COPD), drugs, drug charts, \\ emergency, morbidity, mortality, oxygen, respiratory. \\ Citation: \\ EMJ. 2021; DOI/10.33590/emj/20-00135
}

\section{Abstract}

Oxygen is one of the most commonly used yet poorly prescribed drugs. The 2015 British Thoracic Society (BTS) emergency oxygen audit highlighted the national shortcomings in oxygen prescribing and administration. A 2017 local audit at the Royal Sussex County Hospital, Brighton, UK, continued to demonstrate poor compliance with the BTS Oxygen Prescribing Guidelines in all areas audited. This study carried out yearly reaudits in November 2018 and 2019 to objectively measure the impact of implementing trust-wide and local interventions (July 2018 and August 2019).

Intervention 1 included introduction of the National Early Warning Score (NEWS2) scale and redesigning drug charts with tick-boxes for target oxygen saturations. Intervention 2 included mandatory junior doctor teaching on safe oxygen prescribing, 'oxygen safety' posters on audited wards, and reminders at handover for staff to measure and document oxygen saturations.

Following Intervention 1, all patients with valid oxygen prescriptions had a specified target saturations range. Intervention 2 ensured all patients had actual saturations within their prescribed target range, and $99 \%$ had oxygen saturations documented with sufficient frequency for their NEWS2 score. These were huge improvements from previous audits, during which a significant proportion of patients were at risk of hypercapnia, and those over- or underoxygenated were left unrecognised for hours. Despite improvements, $14 \%$ of patients continued to use oxygen without valid prescriptions in 2019 , and drug charts were inconsistently signed for during drug rounds.

Although the implemented changes enabled drastic improvements for patient safety and quality in oxygen use, future work should ensure oxygen is always treated as a drug with suitable prescription and documentation.

\section{BACKGROUND}

There have been seven national audits looking at was the 2015 British Thoracic Society (BTS) Audit, which showed shortcomings in the prescription of oxygen, the largest of which oxygen prescribing practices. This subsequently led to the publication of the Emergency Oxygen 
Prescribing Guidelines and set the National Improvement Objectives.

A 2017 audit at an acute tertiary care hospital continued to highlight a failure to meet the expected standards. A reaudit was carried out to determine whether the implementation of trust-wide changes in July 2018, namely the introduction of a National Early Warning Score 2 (NEWS2) and newly designed drug charts, had impacted these behaviours. This study carried out yearly reaudits in November 2018 and 2019 to objectively measure the impact of implementing trustwide and local interventions (in July 2018 and August 2019). The 2018 and 2019 audits aimed to compare current oxygen prescribing and delivery practices at the hospital to standards set by the 2015 BTS National Improvement Objectives, and evaluate changes made since the 2017 audit. Adult inpatients on four wards were audited on a single occasion using a standardised questionnaire validated by the BTS.

\section{INTRODUCTION}

Oxygen is one of the most commonly used yet poorly prescribed drugs. There is a risk that patients may be given too little or too much oxygen, thereby increasing their mortality risk. Following the 2015 BTS National Emergency Oxygen Prescribing Audit and publication of the Emergency Oxygen Prescribing Guidelines, which showed national shortcomings,1,2 a 2017 audit at an acute tertiary care hospital continued to highlight a failure to meet the expected standards for oxygen prescribing and administration.

\section{AIMS}

To compare prescribing and oxygen delivery practices at an acute tertiary care hospital to standards set by the 2015 BTS National Improvement Objectives in yearly audits, and to evaluate improvements in oxygen prescribing from the changes that were implemented following a 2017 local oxygen prescribing audit. Improvements included introduction of the NEWS2 scale, new drug charts with a tick-box for target saturations ranges, educational initiatives for doctors, posters, and face-to-face reminders on oxygen nursing staff.

\section{OBJECTIVES}

\section{Primary Objectives}

Based on the 2015 BTS National Improvement Objectives, ${ }^{1}$ the authors aimed to achieve $95 \%$ of patients using oxygen to have a valid prescription with target saturation range; $100 \%$ of nursing and medical staff to be trained in the safe use of oxygen to local trust/health board oxygen policy; and $90 \%$ of patients using oxygen to have oxygen signed for at the most recent drug round.

\section{Secondary Objectives}

Secondary objectives were to determine the percentage of patients receiving oxygen on four inpatient wards; the percentage of patients receiving oxygen with a valid drug chart prescription; the percentage of patients receiving oxygen with a valid drug chart oxygen prescription who have a target saturation range specified; the percentage of patients for whom oxygen saturations were documented with sufficient frequency according to their NEWS2; the percentage of patients receiving oxygen with a specified target saturation range, who had oxygen saturations actually measured within this range; and the percentage of patients who had oxygen signed for at each drug round.

\section{METHODS}

The audit was applied to adult inpatients on four wards at an acute tertiary care hospital. The wards included the acute medical unit, two respiratory wards, and the coronary care unit. Data were collected by two doctors on a single occasion on all wards in November 2017, November 2018, and November 2019, using two standardised data collection sheets validated by the BTS.,2 The inclusion criteria for this audit was adult inpatients aged 18-75 years admitted to one of the four wards as described above.

There were two parts to the data that were collected. In Part 1 (Figure 1), data were collected from individual patients on each ward. 


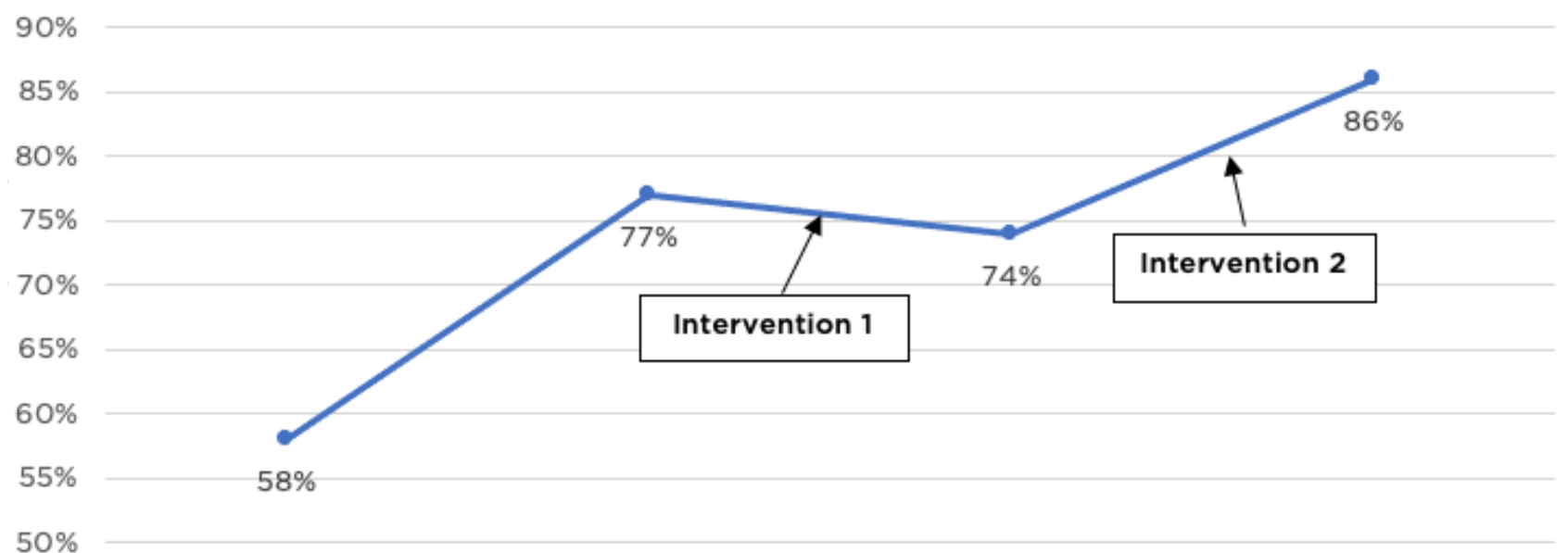

$50 \%$

BTS 2015 National Audit Nov' 2017 Local Audit Nov' 2018 Local Audit Nov' 2019 Local Audit

Figure 1: Percentage of patients using oxygen with a valid drug chart prescription.

This included patient identifier and ward details; oxygen status (whether oxygen was in use); whether a valid oxygen prescription was present; the number of drug rounds that took place in the past 24 hours; the number of times the drug chart was signed for oxygen in the previous 24 hours; the number of times oxygen should have been documented on the NEWS2 chart (based on the patient's score and observation level); the number of times oxygen saturations were actually recorded on the observation chart; and whether the oxygen saturation level of the patient was within their specified target range, and if not how far off.

In Part 2 (Figure 2), data collected from each ward were summarised. This included information on type of ward; date of audit; number of patients on each ward during the audit; number of patients using oxygen with drug chart prescriptions and specified target ranges; the total number of drug rounds in the past 24 hours and signatures on the drug chart for oxygen in the past 24 hours; the total number of observation rounds that should have taken place in the past 24 hours and how many oxygen saturations were actually monitored and recorded on the observation charts; the total number of patients with oxygen saturation levels within their specified target range, and if outside the range how great the deviation from the specified range; and the total number of patients with no oxygen prescription or target range.

Data from the above questionnaires were then collated, summarised, and analysed.

\section{CHANGES IMPLEMENTED}

Trust-wide changes were implemented in July 2018. In Intervention 1, changes included the introduction of NEWS2 where patients with lower target saturations had a dedicated section of the observation chart, which required verification from a registrar or above, and redesigning of the drug chart to include a tickbox specifying target oxygen saturations within the dedicated oxygen prescription section.

Changes were also implemented in AugustOctober 2019. In Intervention 2, changes included mandatory teaching for all junior doctors on safe oxygen prescribing and guidelines; posters containing 'Oxygen is a Drug' and Oxygen Prescribing Guidelines were put up around the audited wards; and reminders were given at handover meetings for nursing staff to measure, document, and administer oxygen appropriately. 


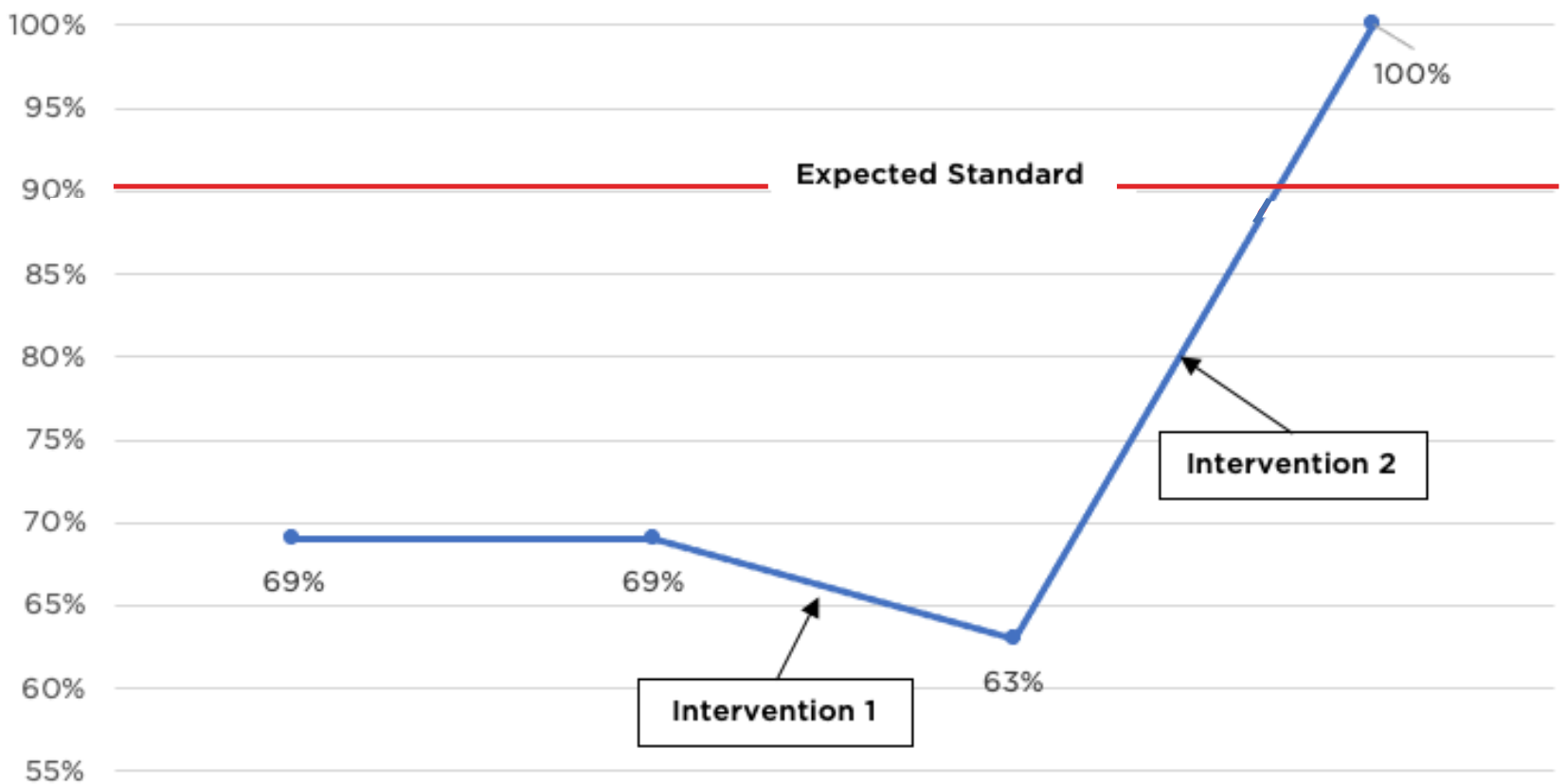

$50 \%$

BTS 2015 National Audit Nov' 2017 Local Audit Nov' 2018 Local Audit Nov' 2019 Local Audit

Figure 2: Percentage of patients with oxygen saturations actually within their specified target saturations range.

\section{RESULTS}

Adult inpatients on four wards (acute medical unit, respiratory ward 1 and respiratory ward 2, and the coronary care unit) between the ages of 18 and 75 years were audited each year. There were 51 patients included in the 2017 audit, 61 patients in the 2018 audit, and 74 patients in the 2019 audit. Among these patients, 33\% were using oxygen in 2017, $23 \%$ in 2018 , and $19 \%$ in 2019.

Results (Table 1) showed that $86 \%$ of patients in the 2019 audit were using oxygen with a valid prescription, relative to $74 \%$ in 2018 and $77 \%$ in 2017 (Figure 1). One hundred percent of patients with a valid drug chart prescription had a target saturations range specified in the 2018 and 2019 audits, compared with $77 \%$ in 2017, which demonstrates a sustainable change and meets the expected standard set by the BTS. Furthermore, 99\% of patients in 2019 had oxygen saturations documented with sufficient frequency for their NEWS2, which represents a 58\% improvement from the 2018 audit (this outcome was not measured in the 2017 audit). There was a $37 \%$ increase (63\% to $100 \%$ ) in patients having actual oxygen saturations within their specified target saturation range in the 2019 reaudit, compared with the 2018 audit. This exceeded the National Improvement Objectives set by the BTS in 2015 (Figure 2). Finally, it appeared that fewer drug charts were being signed for by nursing staff to confirm administration of oxygen in the 2019 audit, relative to 2017 and 2018 .

The study's reaudit in November 2018, following the changes implemented in July 2018 (Intervention 1), found that $26 \%$ of patients were still using oxygen without a valid prescription, $37 \%$ had saturations outside their target range, and only $12 \%$ of drug charts had oxygen signed. However, the new drug charts had ensured that all patients with valid oxygen prescriptions now had target saturations specified, representing a significant improvement from 2017. Whether oxygen saturations were being documented with sufficient frequency as per a patient's NEWS2 was additionally measured. 
Table 1: Comparison of yearly audited parameters with the 2015 British Thoracic Society (BTS) audit results.

\begin{tabular}{|c|c|c|c|c|}
\hline Number of patients (\%) & $\begin{array}{l}2015 \text { BTS } \\
\text { audit }\end{array}$ & $\begin{array}{l}2017 \text { local } \\
\text { audit }\end{array}$ & $\begin{array}{l}2018 \text { local } \\
\text { audit }\end{array}$ & $\begin{array}{l}2019 \text { local } \\
\text { audit }\end{array}$ \\
\hline Patients using oxygen with a valid drug chart prescription & 58 & 77 & 74 & 86 \\
\hline Patients using oxygen with a target saturations range specified & 58 & 77 & 100 & 100 \\
\hline $\begin{array}{l}\text { Patients with oxygen saturations documented with sufficient } \\
\text { frequency for their NEWS2 Score }\end{array}$ & N/A & N/A & 41 & 99 \\
\hline Patients with oxygen saturations within their target saturations range & 69 & 69 & 63 & 100 \\
\hline $\begin{array}{l}\text { Drug charts with a valid oxygen prescription with oxygen signed for } \\
\text { at each drug round }\end{array}$ & 29 & 23 & 12 & 8 \\
\hline
\end{tabular}

BTS: British Thoracic Society; N/A: not applicable; NEWS2: National Early Warning Score 2.

Based on these findings, it was recognised that not all healthcare professionals were treating oxygen as a drug, which requires appropriate prescription, monitoring, and documentation. Further changes were required to alter individual approaches to oxygen. Therefore, changes were implemented as per Intervention 2 above. The 2018 audit and its results were used to educate newly registered junior doctors of the BTS oxygen prescribing guidelines, ${ }^{1,2}$ and the potential dangers if not adhered to, as well as provide face-to-face and poster-based reminders to all healthcare professionals. Although there were improvements in most areas audited, the proportion of valid oxygen prescriptions completed by doctors still did not meet the 95\% expected standards, and drug charts were not being appropriately signed for oxygen at each drug round.

\section{DISCUSSION}

Due to the nature of the drug and its frequent use, unfortunately, oxygen prescribing has proven to be a downfall throughout the cycles for various reasons. Poor compliance with the oxygen prescribing guidelines was found in 2017 in all areas audited. The reaudit in 2018 explored the implementation of trust-wide changes and the impact on oxygen prescribing. In the 2019 audit, $86 \%$ of patients were using oxygen with a valid prescription, compared to $74 \%$ in 2018 and $77 \%$ in 2017. Although there were improvements, the overall picture was disappointing. For an intervention to be effective past its short-term capacity, great focus should be on ongoing input from the staff on the wards in question. ${ }^{3}$

The significance of the implemented changes that were put into place following the 2018 audit enabled drastic improvements. There was a huge increase in the proportion of patients with a valid prescription for oxygen, target saturations range specified, and actual saturations within the specified range. These factors have a direct impact on patient safety and quality of care for those using oxygen in an acute setting. By improving these main areas, patient risk of hypercapnia and hypoxia have been lowered directly. ${ }^{4-6}$

Similar quality improvement initiatives, such as those carried out at a district general hospital in Somerset, UK, to improve compliance with oxygen prescribing guidelines, found that specifically targeting interventions to increase awareness of appropriate oxygen prescribing to the main groups of professionals who use oxygen (nursing staff and junior doctors) can have a significant impact. ${ }^{7}$ Another project $^{3}$ highlighted that teaching sessions for junior and senior doctors and other allied healthcare prescribers can significantly improve the documentation and appropriate prescribing of oxygen. These projects further corroborate the research findings because they both implemented a similar strategy to drive improvement. However, another project $^{9}$ found that poster reminders and multidisciplinary team education only had a 
limited impact on improving oxygen prescribing. It was the use of targeted oxygen stickers above oxygen taps that ultimately enhanced oxygen prescribing practices.

There continues to be challenges around oxygen prescribing and the related consistency with signing of drug charts. ${ }^{4-6,9-11}$ From this study, and previous research in this area, it is has been made apparent that using focussed and targeted interventions is more likely to be able to alter attitudes around documentation and sustain this change. 4,5,12-15 By continuously reinforcing the concept of oxygen being a drug and educating staff, the changes made can not only be sustained but also used trust-wide to positively impact patient outcome. Further targeted interventions, such as oxygen target saturation stickers or wristbands, may lead to even better compliance with the oxygen prescribing guidance and ultimately improve patient safety.

\section{CONCLUSION}

Although multiple changes were implemented over several cycles, the greatest impact was found to be from implementing staff education and awareness. ${ }^{3,7}$ A combination of lack of awareness, not treating oxygen as a drug, and not optimally utilising the drug chart, was exposing patients to harm. Through the amalgamation of organisational changes, and smaller local changes at each hospital, there can be an overall positive impact on patient safety, which can subsequently reduce mortality and morbidity. Simple measures such as staff education, verbal and written reminders, combined with the introduction of systemic changes such as the introduction of new drug charts, and NEWS2 scales have the greatest effect on individual behaviours. Although these trust-wide changes made it easier for oxygen saturation ranges to be documented, it is through local initiatives that each team member can reflect on alterations they could make to their practice on a personal level.

\section{References}

1. O’Driscoll R. British Thoracic Society emergency oxygen audit report. National Respiratory Audit Programme. Annual Report. 2015. Available at: https://www.britthoracic.org.uk/document-library/ quality-improvement/audit-reports/ emergency-oxygen-2015. Last accessed: 29 March 2021.

2. O'Driscoll BR et al. BTS guideline for oxygen use in adults in healthcare and emergency settings. Thorax. 2017;72:i1-90.

3. Shaladi A et al. 53 Audited quality improvement project on oxygen prescribing on an acute surgical ward. BMJ Leader. 2019;3(Suppl 1):A2O.

4. Cousins JL et al. 2016. Acute oxygen therapy: a review of prescribing and delivery practices. Int J Chron Obstruct Pulmon Dis. 2016;11(1):1067-
5. Stoller JK et al. Oxygen therapy for patients with COPD. Chest. 2010:138(1):179-87.

6. Brill SE, Wedzicha JA. Oxygen therapy in acute exacerbations of chronic obstructive pulmonary disease. Int J Chron Obstruct Pulmon Dis. 2014:9:1241-52.

7. Helliar S. Improving oxygen prescribing rates by tailoring interventions for specific healthcare professional groups. BMJ Qual Improv Rep. 2016;5(1):u209520.w4033.

8. Thein OS et al. Oxygen prescription: improving compliance using methods from BMJ Open Quality journal. BMJ Open Qual. 2018;7(2):e000288.

9. Inglis A. Audit of oxygen prescribing Treatment needs to be adjusted. BMJ. 2001;322(7289):799.
10. Goldstein R. 22: The dilemma of oxygen therapy. Respiratory Medicine: COPD Update 2007:3(1):13.

11. Dodd $\mathrm{M}$ et al. Audit of oxygen prescribing before and after the introduction of a prescription chart. BMJ. 2000;321(7265):864-5.

12. Balkissoon R. Journal club: COPD and oxygen therapy. Chron Obstr Pulm Dis. 2017;4(1):71-5

13. Bateman NT, Leach RM. ABC of oxygen. Acute oxygen therapy. BMJ. 1998;317(7161):798-801.

14. COPD Foundation. Oxygen therapy. 2020. Available at: https://www. copdfoundation.org/Learn-More/Iam-a-Person-with-COPD/OxygenTherapy.aspx. Last accessed: 26 May 2020.

15. Why prescribe short-burst oxygen? Drug Ther Bull. 2007:45(9):70-2. 